\title{
Absence of TMPRSS2:ERG fusions and PTEN losses in prostate cancer is associated with a favorable outcome
}

\author{
Maisa Yoshimoto ${ }^{1}$, Anthony M Joshua ${ }^{1}$, Isabela W Cunha ${ }^{2}$, Renata A Coudry ${ }^{2}$, Francisco P \\ Fonseca $^{3}$, Olga Ludkovski ${ }^{1}$, Maria Zielenska ${ }^{4}$, Fernando A Soares ${ }^{2}$ and Jeremy A Squire ${ }^{1,5}$ \\ ${ }^{1}$ Division of Applied Molecular Oncology, Ontario Cancer Institute, Toronto, ON, Canada; ${ }^{2}$ Department of \\ Pathology, Hospital do Câncer, São Paulo, Brazil; ${ }^{3}$ Department of Pelvic Surgery, Hospital do Câncer, São \\ Paulo, Brazil; ${ }^{4}$ Department of Pathology and Laboratory Medicine, The Hospital for Sick Children, Toronto, \\ ON, Canada and ${ }^{5}$ Department of Medical Biophysics, Faculty of Medicine, University of Toronto, Toronto, \\ ON, Canada
}

\begin{abstract}
TMPRSS2:ERG gene fusions and PTEN deletions are the most common genomic aberrations in prostate cancer. Recent work has suggested that the TMPRSS2:ERG fusion is associated with a more aggressive phenotype. Similarly, PTEN deletion has been associated with biochemical recurrence and lymph node metastasis. To date, there has been no systematic analysis of the combined influence of genomic PTEN deletion with TMPRSS2:ERG gene fusions on clinical parameters of prostate cancer progression. We carried out a retrospective analysis of 125 prostate cancers with known clinical outcome using interphase fluorescence in situ hybridization to detect the relative prevalence of TMPRSS2:ERG rearrangements and/or PTEN genomic deletions. TMPRSS2:ERG rearrangement was found in 60 of $125(48 \%)$ prostate cancers. Duplication of TMPRSS2:ERG fusion was observed in seven $(6 \%)$ tumors. Gleason grade $(P=0.0002) / s c o r e(P=0.001)$, median tumor volume $(P=0.0024)$, preoperative PSA $(P=0.001)$ and perineural invasion $(P=0.0304)$ were significantly associated with biochemical recurrence by univariate analysis with TMPRSS2:ERG approaching significance $(P=0.0523)$. By multivariate analysis, relevant factors associated with recurrence were Gleason scores $7(P=0.001)$ and 8-10 $(P=0.015), P T E N$ homozygous deletion $(P=0.013)$ and concurrent TMPRSS2:ERG fusion and PTEN deletion $(P=0.036)$. Kaplan-Meier analysis indicated that the presence of TMPRSS2:ERG fusion was marginally less favorable in comparison to no fusion. Duplication of fusion gene showed worse prognosis. It was possible to determine the relative frequencies of PTEN deletion and/or TMPRSS2:ERG fusions in 82 of 125 prostate cancers. With biochemical recurrence as an endpoint, the genomic biomarkers identified three patient groups: (1) 'poor genomic grade' characterized by both PTEN deletion and TMPRSS2:ERG fusions (23/82, 28\%); (2) 'intermediate genomic grade' with either PTEN deletion or TMPRSS2:ERG fusion (35/82, 43\%) and (3) 'favorable genomic grade' in which neither rearrangement was present $(24 / 82,29 \%)$. Kaplan-Meier and multivariate analysis indicate that TMPRSS2:ERG fusion and PTEN loss together are a predictor of earlier biochemical recurrence of disease.

Modern Pathology (2008) 21, 1451-1460; doi:10.1038/modpathol.2008.96; published online 23 May 2008
\end{abstract}

Keywords: interphase FISH; PTEN haploinsufficiency; prognosis; biomarker; biochemical recurrence

The identification of prognostic molecular biomarkers is recognized as critically important in the future clinical management of prostate cancer. Despite the clinical utility of Gleason score, pathological stage and serum PSA in assessing prognosis and guiding management, further molecular determinants are needed that more accurately address

Correspondence: Dr JA Squire, PhD, Division of Applied Molecular Oncology, Ontario Cancer Institute, Princess Margaret Hospital, 610 University Avenue, Room 9-721, Toronto, ON M5G 2M9 Canada.

E-mail: jeremy.squire@utoronto.ca

Received 14 March 2008; revised 25 April 2008; accepted 26 April 2008; published online 23 May 2008 pathways that underlie tumorigenesis of prostate cancer. ${ }^{1}$

Chromosomal deletions of 10q suggested that the phosphatase and tensin homologue (PTEN) gene at cytoband 10q23.3 is strongly associated with the progression of prostate cancer. ${ }^{2-4}$ PTEN plays an important role in the modulation of the phosphatidylinositol-3-kinase (PI3K) pathway by catalyzing degradation of phosphatidylinositol-(3,4,5)-triphosphate (PIP3) generated by PI3K. ${ }^{5}$ PIP3 activates the protein kinase AKT which then modulates a number of downstream targets with important roles in apoptosis and the cell-cycle progression, including $B A D,{ }^{6}$ CASP 3 and CASP $9,{ }^{7}$ MDM $2,{ }^{8}$ mTOR, ${ }^{9}$ FKHR $^{10}$ and $F O X O 3 A,{ }^{11} p 27^{12}$ and the recently recognized 
JNK pathway. ${ }^{13}$ PTEN deletion is associated with tumor progression ${ }^{14-16}$ and more predictive of shorter time to biochemical recurrence of disease, ${ }^{17}$ emphasizing the crucial role of PTEN as marker of tumor behavior in prostate cancer.

The discovery of recurrent translocations in $\sim 40$ $60 \%$ of prostate carcinoma, ${ }^{4,18-24}$ involving the TMPRSS2 gene at 21q22.3 with members of the erythroblast transformation specific (ETS) transcription factor family, such as ERG (21q22.2), ETV1 (7p21.2), ETV4 (17q21) or ETV5 (3q28) genes, has provided new insights into prostatic carcinogenesis. Although the downstream molecular pathways of ETS fusion genes are only beginning to be clarified, clinical associations have been reported in early studies. ${ }^{19,22,25-28}$ For instance, TMPRSS2:ERG-rearranged prostate cancers have been associated with a greater likelihood of lethal prostate cancer, ${ }^{26,27}$ moderate to poorly differentiated tumors ${ }^{25}$ and higher stage diseases with pelvic lymph node metastases. ${ }^{19}$ Evidence of specific TMPRSS2:ERG isoforms were also described to be associated with high levels of fusion mRNA expression and early biochemical recurrence following radical prostatectomy. ${ }^{22}$ However, there are a number of conflicting studies $^{29,30}$ about the prognostic importance of the gene fusion; so the clinical significance of TMPRSS2:ERG fusions remains to be clarified.

Given the potential of both TMPRSS2:ERG fusions and PTEN genomic deletions to contribute to prognosis prediction in prostate cancer, additional genotype-phenotype correlation studies will be helpful. In addition, it is unclear whether the TMPRSS2:ERG rearrangements may be accompanied by PTEN genomic loss, or in contrast, these are mutually exclusive events. There has been no systematic analysis to date of the frequency at which PTEN and TMPRSS2:ERG rearrangements occur simultaneously in prostate cancer, or to examine clinical phenotypes associated with genomic mutations affecting both pathways. Therefore, this study was designed to retrospectively assess the overall frequency and clinical impact of TMPRSS2:ERG rearrangements using a clinicallyannotated tissue microarray. We also provide the results of clinical impact of cooperation between TMPRSS2:ERG gene rearrangements and PTEN genomic deletion in prostate cancer samples that became available after the publication of our previous study. ${ }^{17}$ Our results further implicate TMPRSS2:ERG rearrangements as a prognostic value in the primary tumor, in addition to providing insights into prostate cancer pathophysiology.

\section{Materials and methods}

\section{Tissue Specimens}

The collection of all tissue specimens, clinical and patient follow-up data was obtained after informed consent in accordance with the Hospital do Câncer
Research Ethics guidelines (São Paulo, Brazil). Archival formalin-fixed, paraffin-embedded tissues were obtained from 125 radical prostatectomies performed between 1997 and 2000 at the Hospital do Câncer, AC Camargo, São Paulo, Brazil. For control purposes, 10 non-neoplastic prostate tissue samples were obtained from patients undergoing surgery solely for benign prostate hyperplasia. The prostate cancer cohort and control specimens were sampled using a $0.6-\mathrm{mm}$ diameter tissue core distributed on tissue microarray slide. Adjacent hematoxylin and eosin (H\&E) stained section was reviewed by two pathologists to determine the presence and extent of morphologically representative areas of the original tumors in each tissue core. Reassessment of Gleason grading in a contiguous H\&E stained tissue microarray section assured the presence of prostate adenocarcinoma and the fidelity of the intended tissue microarray core. The size of tumor was based on assessment of total surface area of gland examined histologically involved by carcinoma. Preoperative PSA level was available for all patients and the PSA nonfailure was defined as PSA remaining below $0.2 \mathrm{ng} / \mathrm{ml}$ after radical prostatectomy.

\section{Fluorescence In Situ Hybridization}

Break-apart fluorescence in situ hybridization (FISH) was used for studying the TMPRSS2:ERG gene rearrangement as previously described. ${ }^{31}$ The following bacterial artificial chromosome (BAC) clones were used. BACs located at: (a) the $3^{\prime} E R G$ gene locus (RP11-476D17, 3'ERG sequence extending inward past exon 4), (b) the $5^{\prime} E R G$ gene locus (RP11-95I21, 5'ERG sequence extending inward into exon 10), (c) the TMPRSS2 locus (RP11-535H11) and (d) the telomeric BACs to the $5^{\prime}$ end of TMPRSS2 locus (RP11-35C4, RP11-891L10 and RP11-260O11, $325 \mathrm{~kb}$ downstream from the $5^{\prime}$ end of the TMPRSS2 gene; Figure 1a). The BAC clones were obtained from The Center for Applied Genomics (Toronto, Canada). DNA was extracted and labeled with SpectrumGreen-dUTP, SpectrumOrange-dUTP (Vysis Inc., Downers Grove, IL, USA) or diethylaminocoumarin (DEAC)-dUTP (PerkinElmer Life and Analytical Sciences, Boston, MA, USA) using the Vysis nick-translation kit according to manufacturer's instructions (Vysis Inc.). The integrity and correct chromosome localization of all BAC clones were verified by hybridization to metaphase spreads of normal peripheral lymphocytes.

Sequential dual-color FISH method was applied to the prostate cancer tissue microarray to investigate the occurrence of PTEN genomic deletion in addition to TMPRSS2:ERG gene rearrangements in 82 of 125 prostate cancer cohort. Dual-color FISH on paraffin-embedded tissue microarray tissue was performed using commercially available DNA probes for cytoband 10q23 (Spectrum 


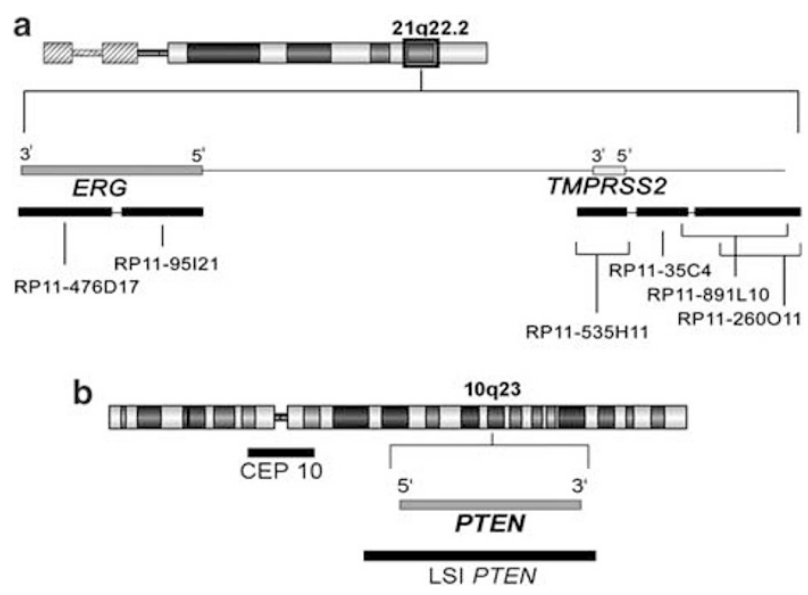

Figure 1 FISH probes used to detect the genomic alteration in prostate cancer. (a) Location and names of the BAC probes spanning the genomic region of the ERG and TMPRSS2 loci at chromosome 21q22.2 and. The linear order and approximate distances of the BAC clones are based on the Human March 2006 assembly of the UCSC Genome Browser. (b) Genomic localization of the commercially available locus-specific PTEN probe and $\alpha$-satellite DNA sequences of chromosome 10 probe (Vysis Inc.).

Orange PTEN locus-specific probe) and region 10p11.1-q11.1 (Spectrum Green centromere of chromosome 10 probe) (LSI PTEN/CEP 10; Vysis Inc.). The PTEN genomic probe spans $368 \mathrm{~kb}$ and starts $166 \mathrm{~kb}$ from $5^{\prime}$ end of the gene and extends $98 \mathrm{~kb}$ past the $3^{\prime}$ end of the gene (Figure 1b). Histologic tissue microarray tissue sections $(5 \mu \mathrm{m})$ were deparaffinized with a series of xylene prior to immersion in $100 \%$ ethanol. FISH was carried out as described. ${ }^{17}$

\section{Data Analysis}

The TMPRSS2:ERG fusion was evaluated for each probe by spot visualization and enumeration in a range from 50 to 100 nonoverlapped, intact interphase nuclei per tumor tissue core using a Zeiss Imager.Z1 microscope equipped with a digital camera AxioCam MRm and AxioVision 4.3 capturing software (Carl Zeiss Canada Ltd, Canada). The $E R G$ rearrangement in tumor nuclei was detected by either the split of one of the colocalized $3^{\prime}$ and $5^{\prime}$ ERG signals in addition to a fused signal of the unaffected chromosome 21 or the hemizygous loss of $5^{\prime}$ ERG (RP11-95I21-green signal), whereas the homologue signal colocalized to the fused signal of the apparently unaffected loci at chromosome 21. The telomeric BACs to the $5^{\prime}$ end of TMPRSS2 locus signal colocalized with the signal of the $3^{\prime}$ ERG BAC, confirming the presence of the typical $5^{\prime}$ TMPRSS2:3' ERG rearrangements. When the BAC telomeric to the $5^{\prime}$ end of TMPRSS2 (blue signal) was well separated from the $3^{\prime} E R G$ BAC (red signal), the TMPRSS2:ERG rearrangement was confirmed using the TMPRSS2 BAC (RP11-535H11-blue), $5^{\prime}$ ERG (RP11-95I21-green) and $3^{\prime}$ ERG BAC
(RP11-476D17-red). Based on hybridization in 10 control cores (data not shown) and tumor cohort, the detection of TMPRSS2:ERG rearrangement was defined as cutoff $>10 \%$ when the distance between signals was $>3$ times the estimated signal diameter. ${ }^{32}$

PTEN copy number was evaluated for each probe by counting spots in a range from 50 to 100 nonoverlapped, intact interphase nuclei per tumor tissue core. Based on hybridization in 10 control cores (data not shown), hemizygous deletion of $P T E N$ were defined as $>20 \%$ (mean +3 s.d. in nonneoplastic controls) of tumor nuclei containing one PTEN locus signal and by the presence of CEP 10 signals. Homozygous deletion of PTEN was exhibited by the simultaneous lack of the both PTEN locus signals and by the presence of control signals $^{32-35}$ in $>30 \%$ of cells. ${ }^{33}$

\section{Statistical Analysis}

FISH findings for TMPRSS2:ERG fusion were correlated in a univariate and multivariate fashion with clinical and pathologic measures of disease aggressiveness. A comprehensive description of the clinical parameters associated with the adenocarcinomas having the TMPRSS2:ERG fusion is summarized in Table 1. Frequency of TMPRSS2:ERG rearrangements was also correlated with PTEN genomic deletion in addition to determinants of disease mortality and morbidity, such as PSA, and extraprostatic extension and time to biochemical relapse. Univariate and multivariate analyses of risk of biochemical failure were studied by Cox proportional hazard model. A significant correlation between two parameters was taken at the 95\% confidence interval. $P$-values $<0.05$ were considered significant. The survival rate was estimated by applying the Kaplan-Meier method. The endpoint for calculating the survival time was defined by time from radical prostatectomy until the occurrence of metastasis or PSA determined biochemical recurrence, ie the date of first PSA increase above $0.2 \mathrm{ng} /$ ml. (median follow-up time 87.4 months, range 11.5-161.6). All calculations were performed using Stata 9.1 (StataCorp LP).

\section{Results}

\section{TMPRSS2:ERG Fusion}

A total of 125 archival tissues with anonymous clinical annotation were analyzed for TMPRSS2:ERG rearrangements by interphase tricolor FISH. TMPRSS2:ERG rearrangement was found in 60 of 125 (48\%) prostatic adenocarcinomas samples. There was evidence of TMPRSS2:ERG fusion with deletion of $5^{\prime} E R G$ probe in 43 of 125 $(34 \%)$ cases. As previously characterized, ${ }^{31}$ sequential FISH analysis using the BAC set telomeric to the $5^{\prime}$ end of TMPRSS2 (RP11-35C4, RP11-891L10 and 
Table 1 Clinicopathological parameters from 122 of the 125 prostatic adenocarcinoma patients

\begin{tabular}{|c|c|c|c|c|}
\hline \multirow[t]{2}{*}{ Clinicopathological parameters } & \multirow[t]{2}{*}{ Number of cases } & \multicolumn{2}{|c|}{ TMPRSS2:ERG } & \multirow[t]{2}{*}{ P-value } \\
\hline & & Not fused & Fusion & \\
\hline Preoperative PSA $(\mathrm{ng} / \mathrm{ml})^{\mathrm{a}}$ & & & & 0.612 \\
\hline $0.9-4.0$ & 9 & $3(33.33)$ & $6(66.67)$ & \\
\hline $4.0-10.0$ & 58 & $31(53.45)$ & $27(46.55)$ & \\
\hline $10.1-20.0$ & 37 & $21(56.76)$ & $16(43.24)$ & \\
\hline $20.1-84.0$ & 15 & $7(46.67)$ & $8(53.33)$ & \\
\hline Median tumor volume (\%) & & & & 0.886 \\
\hline $0-10.0$ & 35 & $19(54.29)$ & $16(45.71)$ & \\
\hline $10.1-20.0$ & 28 & $14(50.00)$ & $14(50.00)$ & \\
\hline $20.1-85.0$ & 49 & $24(48.98)$ & $25(51.02)$ & \\
\hline Gleason score & & & & 0.636 \\
\hline $4-6$ & 74 & $39(52.70)$ & $35(47.30)$ & \\
\hline 7 & 35 & $18(51.43)$ & $17(48.57)$ & \\
\hline $8-9$ & 13 & $5(38.46)$ & $8(61.54)$ & \\
\hline Pathologic stage & & & & 0.966 \\
\hline pT2a & 10 & $6(60.00)$ & $4(40.00)$ & \\
\hline pT2b & 58 & $30(51.72)$ & $28(48.28)$ & \\
\hline pT3a & 39 & $19(48.72)$ & $20(51.28)$ & \\
\hline pT3b & 9 & $4(44.44)$ & $5(55.56)$ & \\
\hline pT4 & 6 & $3(50.00)$ & $3(50.00)$ & \\
\hline Seminal vesicle invasion ${ }^{\mathrm{a}}$ & & & & 0.337 \\
\hline Negative & 108 & $54(50.00)$ & $54(50.00)$ & \\
\hline Positive & 9 & $6(66.67)$ & $3(33.33)$ & \\
\hline Perineural infiltration & & & & 0.344 \\
\hline Negative & 18 & $11(61.11)$ & 7 (38.89) & \\
\hline Positive & 104 & $51(49.04)$ & $53(50.96)$ & \\
\hline Angiolymphatic invasion ${ }^{\mathrm{a}}$ & & & & 0.400 \\
\hline Negative & 89 & $43(48.31)$ & $46(80.70)$ & \\
\hline Positive & 26 & $15(57.69)$ & $11(19.30)$ & \\
\hline Capsular invasion $^{\mathrm{a}}$ & & & & 0.387 \\
\hline Negative & 52 & $29(55.77)$ & $23(44.23)$ & \\
\hline Positive & 69 & $33(47.83)$ & $36(52.17)$ & \\
\hline Extraprostatic extension ${ }^{\mathrm{a}}$ & & & & 0.835 \\
\hline Negative & 92 & $46(50.00)$ & $50(50.00)$ & \\
\hline Positive & 19 & $9(47.37)$ & $10(52.63)$ & \\
\hline Lymphonodal invasion ${ }^{\mathrm{a}}$ & & & & 0.154 \\
\hline Negative & 108 & $53(49.07)$ & $55(50.93)$ & \\
\hline Positive & 2 & $2(100.00)$ & $0(0.00)$ & \\
\hline Biochemical recurrence & & & & 0.047 \\
\hline Negative & 62 & $37(59.68)$ & $25(40.32)$ & \\
\hline Positive & 60 & $25(41.67)$ & $35(58.33)$ & \\
\hline
\end{tabular}

${ }^{\mathrm{a}}$ Values not available for all 122 cases.

The three atypical cases were excluded from the clinicopathological correlation analysis given that complex genomic rearrangement involving an unknown chromosomal partner(s) could not be elucidated as previously described.

Median overall survival was 87.4 months (11.5-161.6).

Values in parentheses indicate the percentage of sample in each category.

$P$-value $=\chi^{2}$-analysis.

RP11-260011-blue) identified a split of the typical colocalized $5^{\prime}$ end of TMPRSS2:3' ERG probe signals in 3 of these 43 samples. This is indicative of a more complex genomic alteration, involving an unknown chromosomal partner(s). Confirmation of the TMPRSS2:ERG fusion in these three samples showing the atypical FISH pattern was obtained by the
BAC set consisting of the $3^{\prime}$ ERG BAC (RP11476D17), 5' ERG (RP11-95I21) and the TMPRSS2 locus (RP11-535H11). Interestingly, an extra copy of TMPRSS2:ERG fusion associated with deletion of $5^{\prime}$ $E R G$ probe was observed in 7 of the 43 samples showing TMPRSS2:ERG fusion with deletion of $5^{\prime}$ ERG (Table 2). There was evidence of FISH 
Table 2 Summary of the TMPRSS2:ERG fusion status by tri-color FISH

\begin{tabular}{l} 
TMPRSS2:ERG status \\
\hline TMPRSS2:ERG fusion via \\
translocation \\
TMPRSS2:ERG fusion via \\
genomic deletion of 5' ERG \\
Other rearrangements \\
Not fused \\
Total
\end{tabular}

Number of cases (\%)

${ }^{\mathrm{a}}$ Duplication of TMPRSS2:ERG fusion was observed in 7 of the 43 samples showing TMPRSS2:ERG fusion via genomic deletion of the $5^{\prime}$ end of the $E R G$ gene.

TMPRSS2:ERG fusion with no deletion of $5^{\prime}$ ERG probe in 17 of $125(14 \%)$ cases. Only 1 of the 17 cases showed the BAC set telomeric to the $5^{\prime}$ end of TMPRSS2 (RP11-35C4, RP11-891L10 and RP11260011-blue) well separated from the $3^{\prime} E R G$ BAC (RP11-476D17-red) (Table 2).

In addition to the 60 TMPRSS2:ERG fused cases, 3 atypical cases had abnormal TMPRSS2:ERG FISH colocalization pattern. The $3^{\prime}$ ERG RP11-476D17 (red signal) did remain juxtaposed to the $5^{\prime} E R G$ RP11-95I21 (green signal), but failed to exhibit the expected colocalization of the TMPRSS2 locus (RP11-535H11-blue) with the ERG gene probes. We were not able to detect FISH fusions between TMPRSS2 and ETV1 or ETV4 in any of these three cases with abnormal FISH TMPRSS2:ERG breakapart results. Furthermore, extra copies of the TMPRSS2 locus (RP11-535H11-blue) were observed in the atypical samples. Such findings indicate that: (a) fusion events between TMPRSS2 and other genes are possible and (b) TMPRSS2:ERG fusions may sometimes have concurrent complex genomic rearrangements within the $\sim 2.9 \mathrm{Mb}$ that separates these two genes. However, the three atypical cases were excluded from the clinicopathological correlation analysis given that complex genomic rearrangement involving an unknown chromosomal partner(s) could not be elucidated.

Among the 60 rearranged TMPRSS2:ERG tumors detected, the Gleason scores were 4-6 (35 tumors), 7 (17 tumors) and 8-9 (8 tumors). A median tumor volume of $>20 \%$ was found in 25/60 rearranged TMPRSS2:ERG tumors. In addition, early biochemical recurrence was detected in all seven samples with an extra copy of TMPRSS2:ERG fusion associated with deletion of $5^{\prime} E R G$ probe.

\section{Concomitant Presence of TMPRSS2:ERG Fusion and PTEN Genomic Deletion}

After acquisition of FISH data, the cases were reviewed to search for potential associations
Table 3 Distribution of samples showing TMPRSS2:ERG fusion and PTEN deletion in 82 prostatic adenocarcinomas

\begin{tabular}{lcc}
\hline & TMPRSS2:ERG fusion & No fusion \\
\hline PTEN deletion & $23(28 \%)$ & $14(17 \%)$ \\
PTEN not deleted & $21(26 \%)$ & $24(29 \%)$ \\
\hline
\end{tabular}

between PTEN deletion as studied previously, ${ }^{17}$ and TMPRSS2:ERG rearrangements. Therefore, we examined differential status of PTEN (deleted or not deleted) and presence of TMPRSS2:ERG fusion in 82 of the 125 tumor samples to assess its utility as biomarker of prognosis. Overall, PTEN deletion in addition to the presence of TMPRSS2:ERG rearrangement was observed in 23 of $82(28 \%)$ prostatic adenocarcinomas. PTEN deletion was also found in 14 prostate adenocarcinoma samples showing absence of TMPRSS2:ERG rearrangement (14/82, $17 \%)$. There was evidence of no copy change of PTEN locus with TMPRSS2:ERG fusion in 21 of 82 (26\%) cases and no copy change of PTEN locus with absence of TMPRSS2:ERG fusion in 24 of $82(29 \%)$ cases. The description of the TMPRSS2:ERG fusion concomitantly with PTEN deletion is summarized in Table 3. A comprehensive description of the clinicopathological parameters associated with 47 adenocarcinomas (23 cases showing both PTEN deletion and TMPRSS2:ERG fusion, and 24 cases in which neither rearrangement was present) is summarized in Table 4. Representative images of TMPRSS2:ERG rearrangement and PTEN deletion are shown in Figure 2.

\section{Statistical Analysis of Clinical Parameters and Genomic Alterations}

High Gleason score and clinical parameters of aggressive disease such as extraprostatic extension $(P=0.0002)$, seminal vesicle invasion $(P=0.0023)$, margin status $(P=0.0008)$, neoadjuvant hormone therapy $(P=0.0004)$, Gleason grade $(P=0.0002) /$ score $(P=0.001)$, median tumor volume $(P=0.0024)$, preoperative PSA $(P=0.001), P T E N$ deletion $(P=0.009)$, concurrent TMPRSS2:ERG fusion and PTEN deletion (0.001) and perineural invasion $(P=0.0304)$ were significantly associated with biochemical recurrence by univariate analysis with TMPRSS2:ERG approaching significance $(P=0.0523$; Table 5). By multivariate analysis, relevant factors to explain biochemical failure included Gleason score (7 and 8-10, $P=0.001$ and $P=0.015$, respectively), concurrent TMPRSS2:ERG fusion and PTEN deletion (0.036) and PTEN homozygous deletion (0.013). The PTEN findings reflect those previously reported for this cohort ${ }^{17}$ (Table 6). For comparison purpose, Kaplan-Meier survival analysis applying established clinical markers, such 
Table 4 Clinicopathological parameters from 47 of the 82 prostatic adenocarcinoma patients analyzed for the differential status of PTEN (deleted or not deleted) and presence of TMPRSS2:ERG fusions

\begin{tabular}{|c|c|c|c|}
\hline & TMPRSS2:ERG fusion and PTEN deletion & TMPRSS2:ERG not fused and PTEN not deleted & $\mathrm{P}$-value \\
\hline Preoperative PSA $(\mathrm{ng} / \mathrm{ml})^{\mathrm{a}}$ & & & 0.638 \\
\hline $0.9-4.0$ & 1 & 0 & \\
\hline $4.0-10.0$ & 10 & 14 & \\
\hline $10.1-20.0$ & 6 & 7 & \\
\hline $20.1-84.0$ & 4 & 3 & \\
\hline Median tumor volume (\%) & & & 0.309 \\
\hline $0-10.0$ & 4 & 10 & \\
\hline $10.1-20.0$ & 5 & 6 & \\
\hline $20.1-85.0$ & 13 & 11 & \\
\hline Gleason score & & & 0.308 \\
\hline $4-6$ & 12 & 16 & \\
\hline 7 & 7 & 7 & \\
\hline $8-9$ & 4 & 1 & \\
\hline Pathologic stage & & & 0.743 \\
\hline pT2a & 1 & 1 & \\
\hline $\mathrm{pT} 2 \mathrm{~b}$ & 12 & 11 & \\
\hline pT3a & 6 & 9 & \\
\hline pT3b & 3 & 1 & \\
\hline pT4 & 1 & 2 & \\
\hline Seminal vesicle invasion ${ }^{\mathrm{a}}$ & & & 0.847 \\
\hline Negative & 18 & 22 & \\
\hline Positive & 2 & 2 & \\
\hline Perineural infiltration & & & 0.242 \\
\hline Negative & 2 & 5 & \\
\hline Positive & 21 & 19 & \\
\hline Angiolymphatic invasion ${ }^{\mathrm{a}}$ & & & 0.129 \\
\hline Negative & 17 & 17 & \\
\hline Positive & 5 & 5 & \\
\hline Extraprostatic extension & & & 0.587 \\
\hline Negative & 17 & 16 & \\
\hline Positive & 6 & 8 & \\
\hline Lymphonodal invasion $^{\mathrm{a}}$ & & & 0.947 \\
\hline Negative & 21 & 20 & \\
\hline Positive & 1 & 1 & \\
\hline
\end{tabular}

${ }^{\mathrm{a}}$ Values not available for all 47 samples.

Median overall survival was 109 months (49.3-161.3).

$P$-value $=\chi^{2}$-analysis

as the preoperative PSA, seminal vesicle invasion and surgical margins status, was considered to identify subgroups with different prognosis with respect to time to relapse after surgery. The estimated disease-free survival curves demonstrated association between TMPRSS2:ERG fusion and short time based on PSA recurrence intervals (Figure 3a). Significantly, the occurrence of duplication of the TMPRSS2:ERG fusion was associated with a much earlier onset of biochemical recurrence based on PSA values (Figure 3b). Furthermore, the estimated disease-free survival curves demonstrated considerable association of coexisting PTEN deletion and TMPRSS2:ERG rearrangements with short time based on PSA recurrence intervals (Figure 3c). This analysis allowed for three broad groupings of differential patient outcome based on time to biochemical recurrence: (1) a poor prognostic group characterized by both PTEN deletion and TMPRSS2:ERG fusions; (2) an intermediate group with either PTEN deletion or TMPRSS2:ERG fusion and (3) a favorable prognostic group with neither event.

\section{Discussion}

The search for accurate biomarkers in prostate cancer is critical for evolution of accurate management of prostate cancer. This study evaluated the two leading genomic biomarkers (PTEN deletion and TMPRSS2:ERG rearrangements) for their contribution to prostate cancer prognosis in a large Brazilian cohort. Figure 3c illustrates the two 

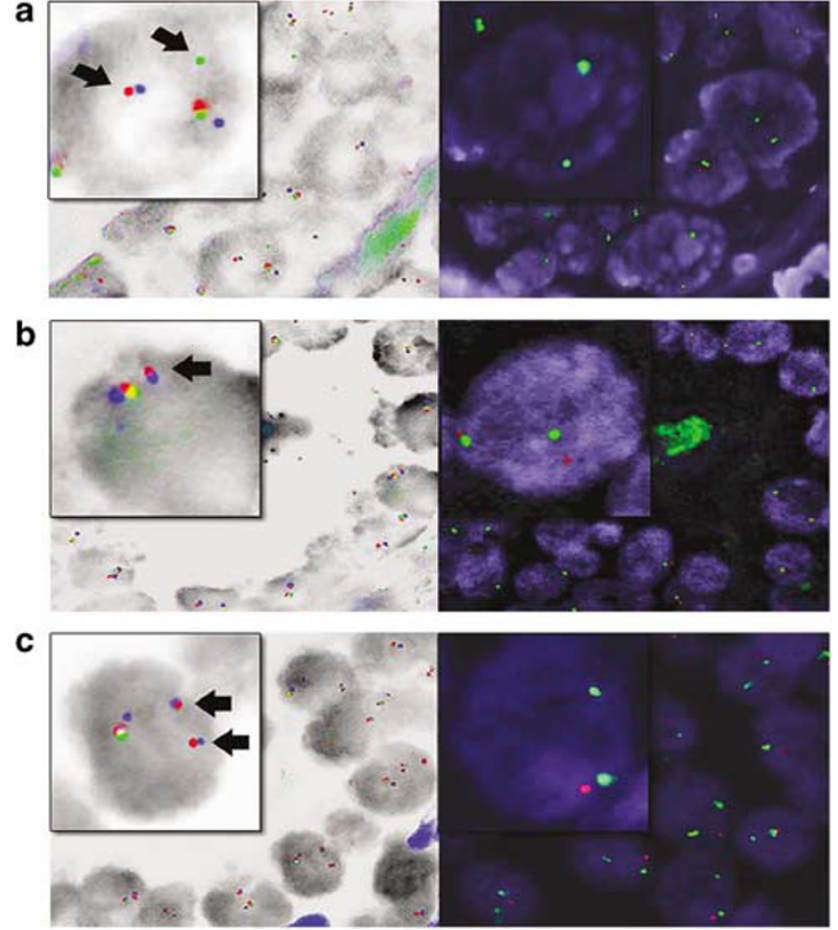

Figure 2 Different patterns of genomic alterations detected by interphase FISH in prostate cancer. (a-c) Representative FISH images are shown for prostate cancer tissue microarray applying the TMPRSS2:ERG (left panel) and PTEN (right panel) probes. The left panel shows a representative pseudo-color image with the inverted-DAPI counterstained nuclei as a gray tone overlay to facilitate interpretation. The right panel shows a representative pseudo-color image with the DAPI counterstained nuclei. The rectangles show a enlarged nucleus FISH image. (a) Representative break-apart tri-color FISH strategy identifies the $5^{\prime} E R G$ BAC (RP11-95I21-green) well separated from the $3^{\prime} E R G$ BAC (RP11-476D17-red) (arrow). The fused signal of the $3^{\prime} E R G$ (red) and TMPRSS2 gene locus (blue) that confirms the TMPRSS2:ERG fusion is arrowed used to detect TMPRSS2:ERG fusions (arrows). The BAC probes hybridizing to the unaffected TMPRSS2:ERG locus show the normal red-green (yellow) and blue colocalization pattern. This extreme nuclear separation is indicative of TMPRSS2:ERG rearrangements via genomic translocation mechanisms. Representative PTEN FISH image of homozygous deletion in prostate cancer shows absence of red signal for 10q23/PTEN locus in most of the nuclei and retained green signals for CEP 10. (b) The fused signal of the $3^{\prime} E R G$ (red) and TMPRSS2 gene locus (blue) that confirms the TMPRSS2:ERG fusion is arrowed. In addition, the hemizygous loss of $5^{\prime} E R G$ BAC (green) indicates an intervening genomic microdeletion of chromosome 21. The BAC probes hybridizing to the unaffected TMPRSS:ERG locus show the normal tri-color (red, green and blue) colocalization pattern. Representative PTEN FISH image of two signals of both red signals (10q23/PTEN locus) and green signals (CEP 10) in most of the nuclei indicating no deletion of PTEN in tumor cells. (c) Duplication of the fused signal of the $3^{\prime} E R G$ (red) and TMPRSS2 gene locus (blue) that confirms the TMPRSS2:ERG fusion is arrowed. In addition, the hemizygous loss of $5^{\prime} E R G$ BAC (green) indicates an intervening genomic microdeletion of chromosome 21. The BAC probes hybridizing to the unaffected TMPRSS:ERG locus show the normal tri-color (red, green and blue) colocalization pattern. The PTEN FISH image shows tumor cells with single red signal for 10q23/PTEN locus in most of the nuclei and paired green signals for CEP 10 indicating hemizygous deletion of 10q23/PTEN locus in prostate cancer. genomic biomarkers (TMPRSS2:ERG fusion and PTEN deletion) which appeared to segregate prostate cancer cases into three broad groupings based on biochemical recurrence as an endpoint: (1) 'poor genomic grade' characterized by both PTEN deletion and TMPRSS2:ERG fusions; (2) 'intermediate genomic grade' with either PTEN deletion or TMPRSS2:ERG fusion and (3) 'favorable genomic grade' in which neither rearrangement was present. Our findings reinforce previous studies that examined each of these promising biomarkers individually and add hypotheses about their interactions in prostatic carcinogenesis.

The proportion of prostatic adenocarcinoma samples with TMPRSS2:ERG rearrangement (48\%) is in agreement with similar cohorts enclosing diverse stage and Gleason scores ${ }^{19,22}$ whereas subsets with earlier stage disease tend to have lower incidence. $^{26,27}$ This association of the gene rearrangement with more advanced disease has been suggested previously. ${ }^{36}$ Indeed several large cohorts have suggested an adverse prognostic impact of the ETS fusion genes with both pathological ${ }^{19,28}$ and overall survival endpoints. ${ }^{26,27}$ The confirmation of the finding of poorest prognosis associated with the duplication of gene fusion via $5^{\prime} E R G$ deletion variant also likely highlight important aspects of the pathophysiology of prostate cancer. The effective double dose of $E R G$ gene alteration associated with this variant is likely to lead to an increased rate of biochemical recurrence seen in our and other cohorts. ${ }^{26,37,38}$ Collectively, our data indicates that the duplication of gene fusion via $5^{\prime} E R G$ deletion variant is predictive of a shorter time to biochemical recurrence of disease. However, some authors have suggested that duplication of the fusion may be a manifestation of general polyploidy rather than a specific duplication event. ${ }^{39}$

As PTEN deletion and TMPRSS2:ERG abnormalities could be additive or mutually exclusive, we evaluated the prognostic information gained by TMPRSS2:ERG analysis alone and in combination with genomic PTEN deletions. The additive effect seen may relate to increased cellular motility, a phenotype that can be attributable to both ETS fusion $^{40}$ and PTEN deletion. ${ }^{41,42}$ Thus together, activation of these pathways may facilitate epithelial-mesenchymal transition that is characteristic of malignant transformation. ${ }^{43}$

To further explore the potential for synergy between these genomic events, we used Oncomine ${ }^{44}$ to interrogate two publicly available microarray studies of prostate cancer progression for differentially expressed genes between ETS overexpressing and nonoverexpressing prostate cancers. ${ }^{45,46}$ The ETS overexpressing prostate cancers demonstrated dysregulation of genes particularly involved in the Wnt pathway. PTEN deletion and its sequel are also likely to affect the same pathways and synergize with the consequences of ETS-related overexpression. Akt activation is also known to inhibit 
Table 5 Univariate Cox proportional hazard analysis of biochemical failure risks (each variable predictor analyzed separately)

\begin{tabular}{|c|c|c|c|c|c|}
\hline \multirow[t]{2}{*}{ Variables } & \multirow[t]{2}{*}{ Category } & \multicolumn{2}{|c|}{$B R F S$} & \multirow[t]{2}{*}{$H R$} & \multirow[t]{2}{*}{$95 \% C I$} \\
\hline & & 5 years & $\mathrm{P}$-value & & \\
\hline \multirow[t]{2}{*}{ Perineural invasion } & Negative & 77.04 & 0.0304 & 1.0 & Reference \\
\hline & Positive & 49.94 & & 2.91 & $1.05-8.04$ \\
\hline \multirow[t]{2}{*}{ Extraprostatic extension } & Negative & 63.45 & 0.0002 & 1.0 & Reference \\
\hline & Positive & 26.78 & & 3.10 & $1.67-5.76$ \\
\hline \multirow[t]{2}{*}{ Margins } & Negative & 62.99 & 0.0008 & 1.0 & Reference \\
\hline & Positive & 25.70 & & 2.40 & $1.41-4.07$ \\
\hline \multirow[t]{2}{*}{ Seminal vesicle invasion } & Negative & 57.33 & 0.0023 & 1.0 & Reference \\
\hline & Positive & 20.00 & & 3.28 & $1.46-7.36$ \\
\hline \multirow[t]{2}{*}{ Neoadjuvant hormonotherapy } & Negative & 59.49 & 0.0004 & 1.0 & Reference \\
\hline & Positive & 33.33 & & 1.59 & $1.21-2.08$ \\
\hline \multirow{2}{*}{ Primary gleason grade } & $2-3$ & 48.01 & 0.0002 & 1.0 & Reference \\
\hline & 4 & 15.43 & & 1.48 & $1.74-6.95$ \\
\hline \multirow[t]{3}{*}{ Gleason score } & $4-6$ & 69.90 & $<0.001$ & 1.0 & Reference \\
\hline & 7 & 32.58 & & 3.14 & $1,79-5.49$ \\
\hline & 8 & 19.94 & & 3.42 & $1.63-7.16$ \\
\hline \multirow[t]{3}{*}{ Median tumor volume } & $0-10.0$ & 71.43 & 0.0024 & 1.0 & Reference \\
\hline & $10.1-20.0$ & 62.95 & & 1.10 & $0.46-2.60$ \\
\hline & $20.1-85.0$ & 39.63 & & 2.67 & $1.35-5.30$ \\
\hline \multirow[t]{4}{*}{ Preoperative PSA } & $0.9-4.0$ & 77.78 & $<0.001$ & 1.0 & Reference \\
\hline & $4.1-10.0$ & 62.85 & & 2.38 & $0.56-10.08$ \\
\hline & $10.1-20.0$ & 55.52 & & 2.86 & $0.66-12.37$ \\
\hline & $20.1-84.0$ & 6.67 & & 13.08 & $2.93-58.26$ \\
\hline \multirow[t]{2}{*}{ TMPRSS2:ERG fusion } & Negative & 61.77 & 0.0523 & 1.0 & Reference \\
\hline & Positive & 45.72 & & 1.99 & $1.21-3.27$ \\
\hline \multirow[t]{3}{*}{ PTEN deletion } & Negative & 57.04 & 0.0009 & 1.0 & Reference \\
\hline & Hemizygous & 50.00 & & 1.53 & $0.82-2.85$ \\
\hline & Homozygous & 0.0 & & 5.93 & $2.12-16.84$ \\
\hline PTEN deletion and & Negative & 59.35 & 0.001 & 1.0 & Reference \\
\hline TMPRSS2:ERG fusion & Positive & 30.43 & & 2.49 & $1.43-4.35$ \\
\hline
\end{tabular}

BRFS, biochemical recurrence-free survival; CI, confidence interval; HR, hazard ratio.

Table 6 Multivariate model to biochemical failure risks by Cox logistic regression analysis

\begin{tabular}{|c|c|c|c|c|}
\hline Variables & Category & $H R$ & $\mathrm{P}$-value & $95 \% C I$ \\
\hline \multirow[t]{3}{*}{ Gleason score } & $4-6$ & 1.0 & & Reference \\
\hline & 7 & 3.07 & $<0.001$ & $1.75-5.37$ \\
\hline & $8-10$ & 2.65 & 0.015 & $1.21-5.81$ \\
\hline \multirow[t]{3}{*}{ PTEN status } & Negative & 1.0 & & Reference \\
\hline & Hemizygous deletion & 1.30 & 0.433 & $0.67-2.54$ \\
\hline & Homozygous deletion & 4.43 & 0.013 & $1.36-14.40$ \\
\hline \multirow{2}{*}{$\begin{array}{l}\text { PTEN deletion and } \\
\text { TMPRSS2:ERG fusion }\end{array}$} & Negative & 1.0 & & Reference \\
\hline & Positive & 1.87 & 0.036 & $1.04-3.36$ \\
\hline
\end{tabular}

CI, confidence interval; HR, hazard ratio.

GSK3 $\beta .^{3,47}$ The combination of these two events would theoretically lead to extra translocation of $\beta$-catenin to the nucleus further assisting cellular motility and epithelial to mesenchymal transition. ${ }^{48}$ Additionally, we observed an upregulation of BRAF in ETS overexpressing tumors, such that combined signaling through ERK is also likely to increase cellular migration phenomena.

Our new findings suggests that the subgroup with absence of both TMPRS2:ERG and genomic PTEN alterations may be designated as favorable genomic grade. Kaplan-Meier and multivariate analysis indicates that TMPRSS2:ERG fusion and PTEN loss together are a predictor of earlier biochemical recurrence of disease. The acquisition of the TMPRSS2:ERG fusion and concomitant PTEN deletion at an earlier phase in prostatic oncogenesis appear to be an additional determinant of the phenotype that govern a more aggressive tumor phenotype. Further studies should validate this 

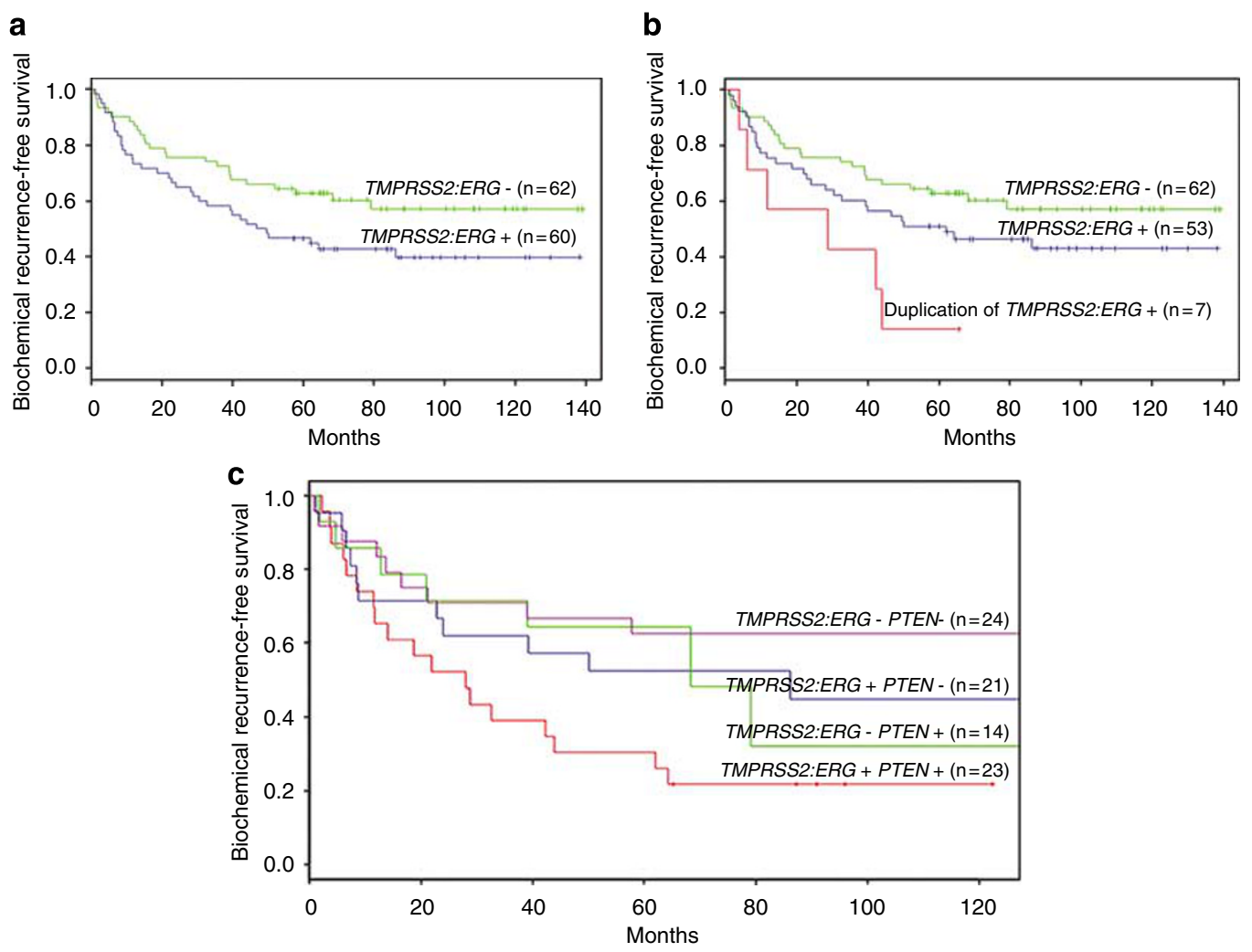

Figure 3 Kaplan-Meier curves illustrating biochemical recurrence-free survival among prostate cancer patients defined by the status of selected clinicopathological parameters, TMPRSS2:ERG rearrangements and PTEN copy number changes; (+) presence, (-) absence. (a) PSA recurrencefree survival curve stratified by the TMPRSS2:ERG rearrangements (absence or presence of gene fusion) on 122 prostate cancer patients and (b) when duplicate TMPRSS2:ERG FISH fusion signals were present (red curve) the outcome was the least favorable. (c) PSA recurrence-free survival analysis stratified by TMPRSS2:ERG rearrangements and PTEN copy number changes on 82 prostate cancer patients.

concept to allow better stratification of care in prostate cancer.

\section{Acknowledgements}

We acknowledge the assistance of Dr Arul Chinnaiyan and Dr Scott Tomlins in our Oncomine data analysis. This work has been supported by the Prostate Cancer Research Foundation of Canada (PCRFC), the National Cancer Institute of Canada (NCIC), the Department of Defense Congressionally Directed Medical Research Program Predoctoral Traineeship Award (PC050531) and the American Urological Association Foundation/ Astellas Research Scholar award.

\section{References}

1 Joshua AM, Evans A, Van der Kwast T, et al. Prostatic preneoplasia and beyond. Biochim Biophys Acta 2008;1785:156-181.

2 Hughes S, Yoshimoto M, Beheshti B, et al. The use of whole genome amplification to study chromosomal changes in prostate cancer: insights into genome-wide signature of preneoplasia associated with cancer progression. BMC Genomics 2006;7:65.

3 Yoshimoto M, Cutz JC, Nuin PA, et al. Interphase FISH analysis of PTEN in histologic sections shows genomic deletions in $68 \%$ of primary prostate cancer and $23 \%$ of high-grade prostatic intra-epithelial neoplasias. Cancer Genet Cytogenet 2006;169:128-137.

4 Yoshimoto M, Joshua AM, Chilton-Macneill S, et al. Three-color FISH analysis of TMPRSS2/ERG fusions in prostate cancer indicates that genomic microdeletion of chromosome 21 is associated with rearrangement. Neoplasia 2006;8:465-469.

5 Besson A, Robbins SM, Yong VW. PTEN/MMAC1/ TEP1 in signal transduction and tumorigenesis. Eur J Biochem 1999;263:605-611.

6 Datta SR, Dudek H, Tao X, et al. Akt phosphorylation of BAD couples survival signals to the cell-intrinsic death machinery. Cell 1997;91:231-241.

7 Cardone MH, Roy N, Stennicke HR, et al. Regulation of cell death protease caspase-9 by phosphorylation. Science 1998;282:1318-1321.

8 Ashcroft M, Ludwig RL, Woods DB, et al. Phosphorylation of HDM2 by Akt. Oncogene 2002;21:1955-1962.

9 Majumder PK, Febbo PG, Bikoff R, et al. mTOR inhibition reverses Akt-dependent prostate intraepithelial neoplasia through regulation of apoptotic and HIF-1dependent pathways. Nat Med 2004;10:594-601.

10 Brunet A, Bonni A, Zigmond MJ, et al. Akt promotes cell survival by phosphorylating and inhibiting a Forkhead transcription factor. Cell 1999;96:857-868.

11 Trotman LC, Alimonti A, Scaglioni PP, et al. Identification of a tumour suppressor network opposing nuclear Akt function. Nature 2006;441:523-527.

12 Graff JR, Konicek BW, McNulty AM, et al. Increased AKT activity contributes to prostate cancer progression 
by dramatically accelerating prostate tumor growth and diminishing p27Kip1 expression. J Biol Chem 2000;275:24500-24505.

13 Vivanco I, Palaskas N, Tran C, et al. Identification of the JNK signaling pathway as a functional target of the tumor suppressor PTEN. Cancer Cell 2007;11:555-569.

14 Koksal IT, Dirice E, Yasar D, et al. The assessment of PTEN tumor suppressor gene in combination with Gleason scoring and serum PSA to evaluate progression of prostate carcinoma. Urol Oncol 2004;22:307-312.

15 Bertram J, Peacock JW, Fazli L, et al. Loss of PTEN is associated with progression to androgen independence. Prostate 2006;66:895-902.

16 Schmitz M, Grignard G, Margue C, et al. Complete loss of PTEN expression as a possible early prognostic marker for prostate cancer metastasis. Int J Cancer 2007;120:1284-1292.

17 Yoshimoto M, Cunha IW, Coudry RA, et al. FISH analysis of 107 prostate cancers shows that PTEN genomic deletion is associated with poor clinical outcome. Br J Cancer 2007;97:678-685.

18 Tomlins SA, Mehra R, Rhodes DR, et al. TMPRSS2:ETV4 gene fusions define a third molecular subtype of prostate cancer. Cancer Res 2006;66: 3396-3400.

19 Perner S, Demichelis F, Beroukhim R, et al. TMPRSS2:ERG fusion-associated deletions provide insight into the heterogeneity of prostate cancer. Cancer Res 2006;66:8337-8341.

20 Cerveira N, Ribeiro FR, Peixoto A, et al. TMPRSS2-ERG gene fusion causing ERG overexpression precedes chromosome copy number changes in prostate carcinomas and paired HGPIN lesions. Neoplasia 2006;8:826-832.

21 Tomlins SA, Rhodes DR, Perner S, et al. Recurrent fusion of TMPRSS2 and ETS transcription factor genes in prostate cancer. Science 2005;310:644-648.

22 Wang J, Cai Y, Ren C, et al. Expression of variant TMPRSS2/ERG fusion messenger RNAs is associated with aggressive prostate cancer. Cancer Res 2006;66:8347-8351.

23 Ahlers CM, Figg WD. ETS-TMPRSS2 fusion gene products in prostate cancer. Cancer Biol Ther 2006;5:254-255.

24 Soller MJ, Isaksson M, Elfving P, et al. Confirmation of the high frequency of the TMPRSS2/ERG fusion gene in prostate cancer. Genes Chromosomes Cancer 2006; 45:717-719.

25 Rajput AB, Miller MA, De Luca A, et al. Frequency of the TMPRSS2:ERG gene fusion is increased in moderate to poorly differentiated prostate cancers. J Clin Pathol 2007;60:1238-1243.

26 Attard G, Clark J, Ambroisine L, et al. Duplication of the fusion of TMPRSS2 to ERG sequences identifies fatal human prostate cancer. Oncogene 2008;27:253-263.

27 Demichelis F, Fall K, Perner S, et al. TMPRSS2:ERG gene fusion associated with lethal prostate cancer in a watchful waiting cohort. Oncogene 2007;26:4596-4599.

28 Nami RK, Sugar L, Wang Z, et al. Expression of TMPRSS2:ERG gene fusion in prostate cancer cells is an important prognostic factor for cancer progression. Cancer Biol Ther 2007;6:40-45.

29 Winnes M, Lissbrant E, Damber JE, et al. Molecular genetic analyses of the TMPRSS2-ERG and TMPRSS2ETV1 gene fusions in 50 cases of prostate cancer. Oncol Rep 2007;17:1033-1036.

30 Petrovics G, Liu A, Shaheduzzaman S, et al. Frequent overexpression of ETS-related gene-1 (ERG1) in prostate cancer transcriptome. Oncogene 2005;24: 3847-3852.
31 Yoshimoto M, Ludkovski O, Bayani J, et al. Microdeletion and concurrent translocation associated with a complex TMPRSS2:ERG prostate cancer gene fusion. Genes Chromosomes Cancer 2007;46:861-863.

32 Ventura RA, Martin-Subero JI, Jones M, et al. FISH analysis for the detection of lymphoma-associated chromosomal abnormalities in routine paraffin-embedded tissue. J Mol Diagn 2006;8:141-151.

33 Korshunov A, Sycheva R, Gorelyshev S, et al. Clinical utility of fluorescence in situ hybridization (FISH) in nonbrainstem glioblastomas of childhood. Mod Pathol 2005;18:1258-1263.

34 Kawai T, Hiroi S, Nakanishi K, et al. Abnormalities in chromosome 17 and p53 in lung carcinoma cells detected by fluorescence in situ hybridization. Pathol Int 2004;54:413-419.

35 Mezzelani A, Alasio L, Bartoli C, et al. c-erbB2/neu gene and chromosome 17 analysis in breast cancer by FISH on archival cytological fine-needle aspirates. Br J Cancer 1999;80:519-525.

36 Mehra R, Tomlins SA, Shen R, et al. Comprehensive assessment of TMPRSS2 and ETS family gene aberrations in clinically localized prostate cancer. Mod Pathol 2007;20:538-544.

37 Tomlins SA, Laxman B, Dhanasekaran SM, et al. Distinct classes of chromosomal rearrangements create oncogenic ETS gene fusions in prostate cancer. Nature 2007;448:595-599.

38 Tomlins SA, Laxman B, Varambally S, et al. Role of the TMPRSS2-ERG gene fusion in prostate cancer. Neoplasia 2008;10:177-188.

39 Gopalan A LM, Satagopan JM, Zhou QC, et al. 97th Annual Meeting of the United States and Canadian Academy of Pathology. The United States and Canadian Academy of Pathology: Denver, Colorado, 2008.

40 Hsu T, Trojanowska M, Watson DK. Ets proteins in biological control and cancer. J Cell Biochem 2004;91:896-903.

41 Shukla S, Maclennan GT, Hartman DJ, et al. Activation of PI3K-Akt signaling pathway promotes prostate cancer cell invasion. Int J Cancer 2007;121:1424-1432.

42 Kotelevets L, van Hengel J, Bruyneel E, et al. The lipid phosphatase activity of PTEN is critical for stabilizing intercellular junctions and reverting invasiveness. J Cell Biol 2001;155:1129-1135.

43 Larue L, Bellacosa A. Epithelial-mesenchymal transition in development and cancer: role of phosphatidylinositol $3^{\prime}$ kinase/AKT pathways. Oncogene 2005;24: 7443-7454.

44 Rhodes DR, Kalyana-Sundaram S, Mahavisno V, et al. Oncomine 3.0: genes, pathways, and networks in a collection of 18000 cancer gene expression profiles. Neoplasia 2007;9:166-180.

45 Lapointe J, Li C, Higgins JP, et al. Gene expression profiling identifies clinically relevant subtypes of prostate cancer. Proc Natl Acad Sci USA 2004;101:811-816.

46 Glinsky GV, Glinskii AB, Stephenson AJ, et al. Gene expression profiling predicts clinical outcome of prostate cancer. J Clin Invest 2004;113:913-923.

47 Maira SM, Galetic I, Brazil DP, et al. Carboxyl-terminal modulator protein (CTMP), a negative regulator of $\mathrm{PKB} / \mathrm{Akt}$ and $\mathrm{v}$-Akt at the plasma membrane. Science 2001;294:374-380.

48 Polette M, Mestdagt M, Bindels S, et al. Beta-catenin and ZO-1: shuttle molecules involved in tumor invasion-associated epithelial-mesenchymal transition processes. Cells Tissues Organs 2007;185:61-65. 